\title{
ATTENUATION OF INITIAL OSCILLATION IN BRIDGE CRANES VIA INPUT-SHAPING-BASED FEEDBACK CONTROL METHODS
}

\author{
Greg J. Mann \\ School of Mechanical Engineering \\ Georgia Institute of Technology \\ Atlanta, Georgia 30313 \\ mann.gregory.j@gmail.com
}

\author{
Pavel Danilochkin \\ School of Mechanical Engineering \\ Georgia Institute of Technology \\ Atlanta, Georgia 30313 \\ pdanilochkin@gmail.com
}

\author{
William E. Singhose* \\ School of Mechanical Engineering \\ Georgia Institute of Technology \\ Atlanta, Georgia 30313 \\ singhose@gatech.edu
}

\section{ABSTRACT}

Crane payloads are frequently subject to oscillations induced through movement and external disturbances. These oscillations degrade performance and can be dangerous. Input shaping is an effective method to move cranes without creating oscillation, however it is not effective on initial swing. This paper develops a feedback control system, with two operation modes based on input shaping, that allows a crane operator to remove initial oscillations while retaining control over the crane movement direction. The system is shown to be simple to implement, and the effectiveness of the two operation modes is verified experimentally on a scale bridge crane.

\section{NOMENCLATURE}

$\theta(t)$

$\zeta$

$\omega_{a}$

$\omega_{d}$

$\omega_{m}$

$\omega_{n}$

$g$

b

\section{$L$}

$L_{a}$

$L_{m}$

$A_{a}$

$A_{i}$

$A_{m}$ angle of deflection from vertical

degrees

$$
\text { damping ratio }
$$

actual frequency of payload oscillation $\mathrm{rad} / \mathrm{sec}$ damped frequency of payload oscillation $\mathrm{rad} / \mathrm{sec}$ measured frequency of payload oscillation $\mathrm{rad} / \mathrm{sec}$ natural frequency of payload oscillation $\mathrm{rad} / \mathrm{sec}$ acceleration due to gravity

damping constant

hoist length

actual hoist length

measured hoist length

actual oscillation amplitude

$\mathrm{i}^{\text {th }}$ impulse amplitude

measured amplitude
$A_{R}$

$T$

$T_{m}$

$m_{p}$

V

$V_{\max }$

$V\left(\omega_{n}, \zeta\right)$

$x$

$x_{a}$

measured by overhead camera

$t_{i} \quad$ time of $i^{\text {th }}$ impulse $c \quad$ actual to measured frequency ratio requested velocity amplitude $\quad \mathrm{m} / \mathrm{s}$ oscillation period $\mathrm{sec}$

measured oscillation period $\mathrm{sec}$ payload mass $\quad k g$

magnitude of step velocity input to trolley $\mathrm{m} / \mathrm{s}$ maximum trolley velocity $\mathrm{m} / \mathrm{s}$

residual vibration amplitude $\quad \mathrm{m} / \mathrm{s}$

linear displacement of payload as measured by overhead camera $\mathrm{m}$ actual linear displacement of payload as time of $i^{\text {th }}$ impulse sec

\section{INTRODUCTION}

Bridge cranes are often used in shipping and manufacturing industries to move heavy objects. An inherent problem with cranes is payload swing induced by the movement of the trolley and external disturbances. Input shaping is an effective and robust method to move payloads without inducing residual vibrations [1]. However, it is generally not possible to cancel payload oscillations with input shaping alone if the system has initial vibration prior to the beginning of motion. These undesirable oscillations can propagate throughout the entire motion execution, making crane operation very difficult and potentially dangerous.

The goal of this research is two-fold: design a simple control system that cancels out initial payload swing, and allow for control over the direction of the cancellation action. To this end, two parallel systems were developed. One system employes a series of velocity pulses to cancel oscillation and the other achieves

*Address all correspondence to this author. 
the same effect by adaptively shaping the start of motion. In this manner, an operator is able to either cancel the initial vibrations and then continue, or remove oscillation while performing the task at hand. The key performance objectives for the control system were to accurately detect the amplitude and frequency of the vibrations, cancel out the initial swing, and allow for directional control.

\section{Previous Work}

Command-shaping control has been applied to several types of cranes [2-9]. Robust input shaping techniques to reduce vibration due to movement were developed by Singer and Seering [10]. A vector diagram based approach to input shaping was introduced by Singhose et al. [11]. These techniques have the capability of reducing payload swing due to crane motion. Further advancements were made to actively reduce oscillations due to external disturbances using feedback. Sorensen et al. [5] developed a PD feedback control system using an overhead camera. Hekman and Singhose [6] developed an on-off control system that uses an overhead camera, measuring the payload swing and generating on-off commands based on amplitude of the payload swing during motion or while stationary. In this paper, a similar adaptive input shaping control system is developed to cancel payload oscillations caused by external disturbances that uses variable magnitude commands rather than on-off control.

\section{SYSTEM AND SYSTEM MODEL}

The methods developed in this paper were designed and tested on the Portable Bridge Crane in the Advanced Crane Control Laboratory at Georgia Tech, shown in Fig. 1. The crane workspace is approximately $1 \times 1 \times 1.6 \mathrm{~m}$. A camera mounted to the trolley of the crane measures payload deflection.

The crane was modeled as a simple pendulum attached to a cart, as shown in Fig. 2. The velocity of the trolley can be controlled directly; however control of payload position is desired. It is therefore necessary to develop a relationship between trolley velocity and payload deflection.

The dynamics of the model can be described by [5]:

$$
\ddot{\theta}+\left(\frac{b}{L m_{p}}\right) \dot{\theta}+\left(\frac{g}{L}\right) \theta=\left(\frac{-1}{L}\right) \ddot{x}
$$

Applying the Laplace transform and rearranging to form a transfer function yields:

$$
\frac{\Theta(s)}{V(s)}=\frac{\left(\omega_{n}^{2} / g\right) s}{s^{2}+2 \zeta \omega_{n} s+\omega_{n}^{2}}
$$

where,

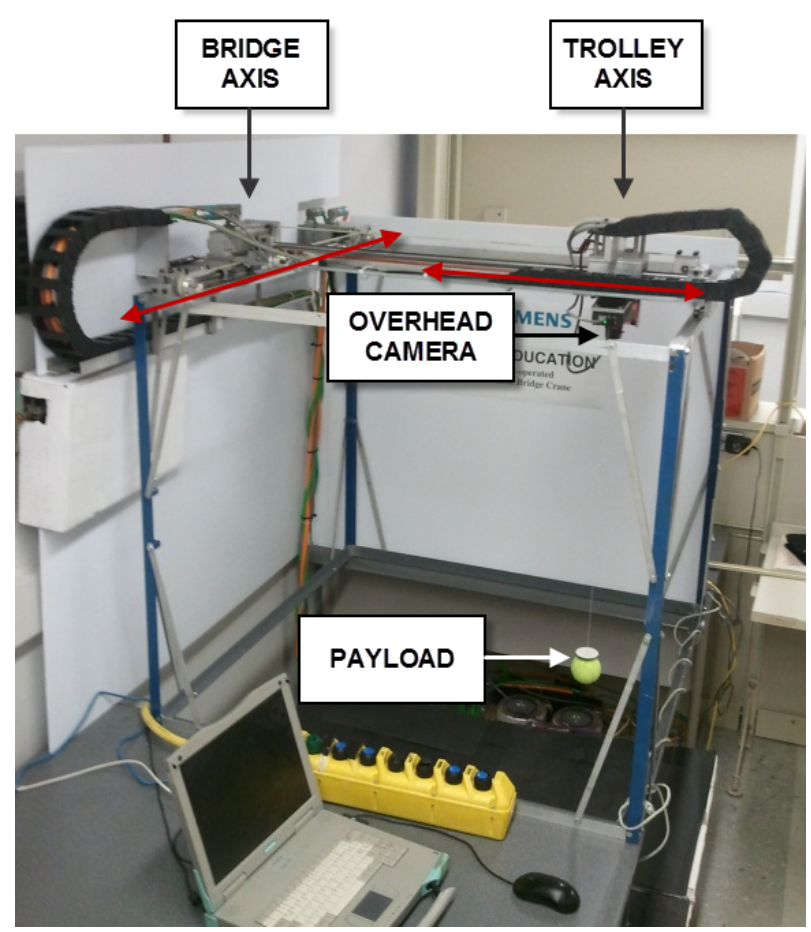

Figure 1. PORTABLE BRIDGE CRANE

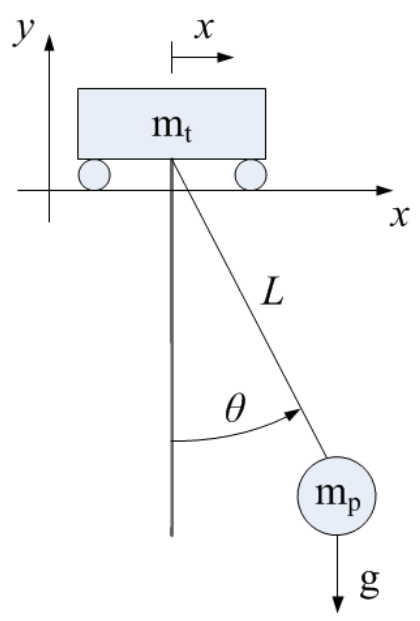

Figure 2. MODEL OF A BRIDGE CRANE [3]

$$
\left(\frac{b}{L m_{p}}\right)=2 \zeta \omega_{n} \quad\left(\frac{g}{L}\right)=\omega_{n}^{2} \quad\left(\frac{-1}{L}\right)=-\frac{\omega_{n}^{2}}{g}
$$

\section{DESIGN OF CONTROL SYSTEM}

The purpose of this research is to create a simple control system that allows an operator to remove payload oscillations while still having control over the movement direction of the crane. To 
this end, two parallel systems were developed. One system creates a pulse in the direction specified to cancel vibration and will be referred to as the pulse mode. The other system shapes the start of the user specified motion to cancel out initial vibration and will be called the move mode.

In order for a feedback control system to cancel motion of a system, it is clear that it must read the state of the system at some point. In the case of this control system, it was decided that no monitoring or calculations would be performed until input from the operator was received. This was, in part, due to the key performance goal for this control system: the ability for the operator to have control over the direction of the cranes movements. Additionally, waiting to retrieve data about the system state allows for much simpler architecture during implementation.

It was assumed that the suspension length of the payload was known. Knowledge of the suspension length allows the frequency of oscillation to be estimated. Additionally, it was assumed that the payload would have some initial oscillation that can be represented as the response from an initial velocity impulse $\left(A_{0}, t_{0}\right)$. Knowing the frequency and thus period of the vibration allows the amplitude and time of the initial impulse to be found from a measured characteristic point in the oscillation. A characteristic point that is easy to measure is the maximum or minimum peak of the oscillation. The maximum or minimum deflection amplitude of a pendulum is approximately a quarter of a period before or after the maximum velocity of the swing. This allows the initial impulse to be placed in time. Including both the maximum and minimum amplitude as points means that one will be found within, at most, half a period.

\section{Pulse Mode}

Once the initial impulse amplitude and timing have been identified, impulses may be placed to cancel the oscillation. The impulses are different for each control mode. In the pulse mode, a positive and a negative impulses are used. Two impulses $\left(A_{1} \& A_{2}\right)$ are used because a velocity limit of the crane constrained the impulse amplitude. Using two impulses doubled the initial swing amplitude that could be canceled. This approach is shown as a vector diagram in Fig. 3 , where impulses $A_{1}$ and $A_{2}$ combine to cancel $A_{0}$. Figure 4 shows the cancelling effect in the time domain.

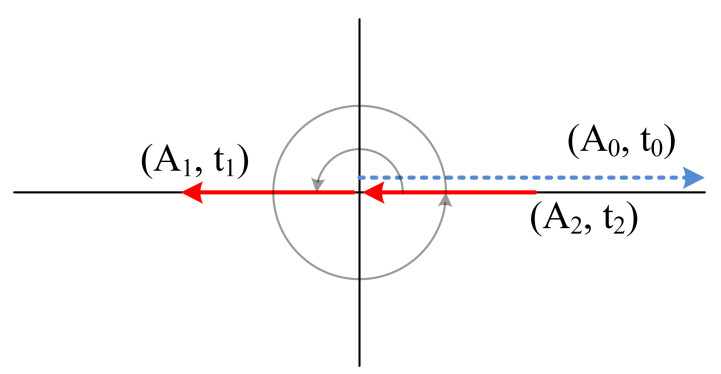

Figure 3. PULSE MODE VECTOR DIAGRAM

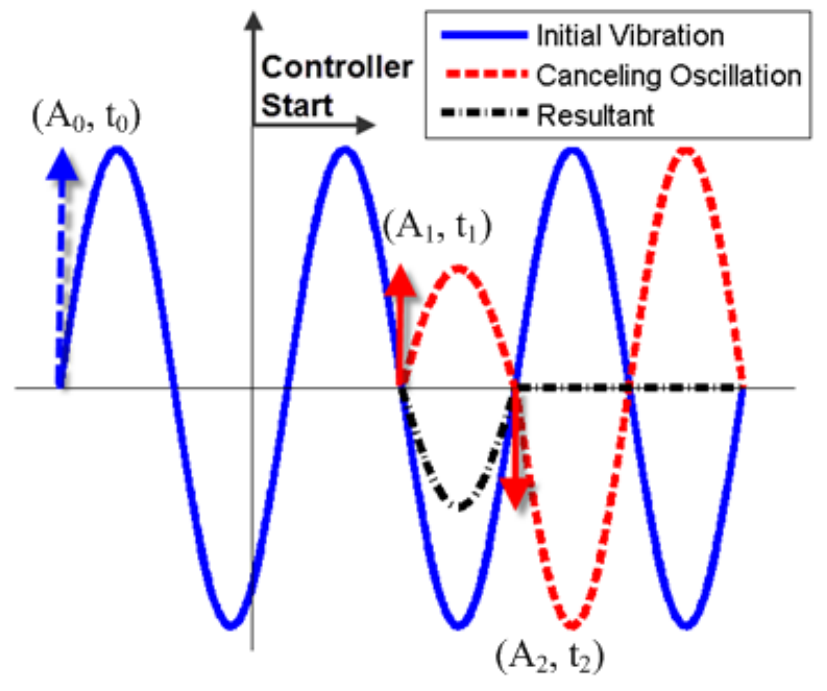

Figure 4. PULSE MODE DEFLECTION PLOT

As shown in the vector diagram, impulse $\left(A_{1}, t_{1}\right)$ must be applied $n / 2$ of a period after the initial impulse where $n$ is an odd integer. The second impulse $\left(A_{2}, t_{2}\right)$ then follows a half of a period later. The amplitudes are related by:

$$
A_{1}=-A_{2}=\frac{1}{2} A_{0}
$$

The orientation of these impulses also depends on the direction the operator wishes to move the crane. Depending on the direction required, $A_{0}$ can be assumed to be positive at a positive zero crossing, or negative at a negative zero crossing.

\section{Move Mode}

The move mode control system is slightly more complex and is similar to the on-off control technique developed by Hekman and Singhose [6]. The system works by adaptively shaping only the start of motion with two positive impulses. The position and size of these impulses varies based on the relationship between the initial impulse amplitude $A_{0}$ and the user requested velocity $A_{R}$. The impulse amplitudes are always equal to half $A_{R}$. The required canceling vibrations must then be created by timing the impulses correctly. This approach is shown on the vector diagram in Fig. 5, where $\theta$ is defined as:

$$
\theta=\cos ^{-1} \frac{A_{0}}{A_{R}}
$$

The impulses converge to a single impulse as $A_{0}$ approaches $A_{R}$. If $A_{0}$ is larger than $A_{R}$, then they are treated as equal. If $A_{0}$ is 


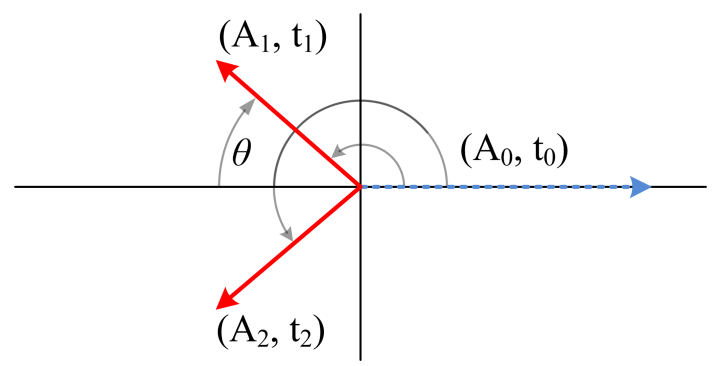

Figure 5. MOVE MODE VECTOR DIAGRAM

small, then the impulses approach the effect of a zero vibration $(\mathrm{ZV})$ shaper [12] aligned along the vertical axis on the vector diagram in Fig. 5. The initial velocity impulse is determined using the maximum deflection which is at phase $\pi / 2$. There is some delay associated with determining the maximum, which means that it is possible for the impulse $\left(A_{1}, t_{1}\right)$ to need to occur before the time of calculation. In this case, the first impulse is delayed by a period and the second impulse becomes the first. The controller impulse amplitudes and timings are:

$$
\left[\begin{array}{c}
A_{i} \\
t_{i}
\end{array}\right]=\left[\begin{array}{cc}
0.5 A_{R} & 0.5 A_{R} \\
\frac{\pi-\theta}{2 \pi} T & \frac{\pi+\theta}{2 \pi} T
\end{array}\right]
$$

where $T$ is the period of the oscillation.

While the start of motion is used to cancel the initial swing, the goal at the end of motion is not to introduce any new swing. The stop is therefore shaped using ZV shaping, and any other input shaping techniques would work as well. The shaper is calculated using the period estimated from the suspension length. The end of motion was not used for vibration canceling so that the move would procede with minimal transient swing and the crane would always stop predictably.

\section{Quick Stop}

In move mode, there is usually time between the two impulses. If the velocity input drops to zero during this time, then the quick stop condition is triggered. This condition involves overlapping the move mode start and a ZV stop. This will result in the crane stopping predictably within half a period of the stop command. This condition is specified separately here because of how it is handled in implementation. The quick stop condition is shown as a vector diagram in Fig. 6 , where $\left(A_{1}, t_{1}\right)$ and $\left(A_{3}\right.$, $\left.t_{3}\right)$ are the two impulses from the move mode start, while $\left(A_{2}\right.$, $\left.t_{2}\right)$ and $\left(A_{4}, t_{4}\right)$ are a $\mathrm{ZV}$ stop that begins when the zero velocity command is given by the operator. The amplitudes of all four impulses are equal.

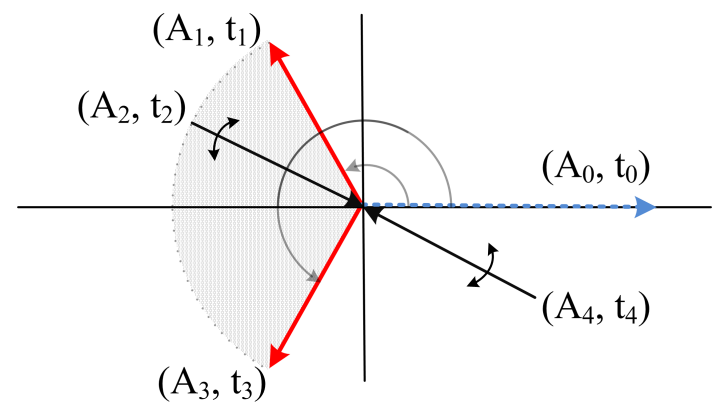

Figure 6. QUICK STOP VECTOR DIAGRAM

\section{Driving the System}

Impulses cannot be used directly to drive the system; instead, they are convolved with a unit step input. This results in the pulse mode creating a pulse in velocity and the move mode creating a shaped velocity profile up to the desired velocity. To control deflection precisely, a relationship between the amplitude of the velocity step and the horizontal deflection amplitude must be found. From Eq. 2, substituting a step velocity input for $V(s)$ and assuming zero damping, we have:

$$
\Theta(s)=\frac{\left(-\omega_{n}^{2} / g\right) s}{s^{2}+\omega_{n}^{2}} \cdot \frac{V}{s}=\frac{\left(-\omega_{n}^{2} / g\right) V}{s^{2}+\omega_{n}^{2}}
$$

Converting from the Laplace domain into the time domain yields:

$$
\theta(t)=\left(\frac{-\omega_{n}^{2}}{g}\right)\left(\frac{V}{\omega_{n}}\right) \sin \left(\omega_{n} t\right)
$$

Also,

$$
L \sin (\theta)=x \rightarrow \theta=\sin ^{-1}\left(\frac{x}{L}\right)
$$

The angular displacement of the payload is converted to a linear displacement in this equation as, in the future, the linear payload displacement will be used for all calculations. This is the measurement provided by the overhead camera. From Eq. 8, the amplitude of oscillation is:

$$
\theta=\left(-\frac{\omega_{n}}{g}\right) V
$$

From (9) and (10), the magnitude of the step velocity input for a payload amplitude $x$ is: 


$$
V=\frac{g}{\omega_{n}} \sin ^{-1}\left(\frac{x}{L}\right)
$$

This equation allows the velocity pulse to be sized correctly. Finally, the bridge crane can only approximate a step. It was necessary to compensate for the slight rise time that was observed in the step response of the trolley. This was accomplished by starting the pulse earlier by approximately the observed rise time.

\section{IMPLEMENTATION OF CONTROL SYSTEM}

A suggested structure for a complete control system implementation is shown in Fig. 7. The payload swing is measured first. This may be accomplished by the operator or automatically. If no initial swing exists, then movement can be completed using normal input shaping techniques. If initial swing exists, then the control system developed here is used. If the system is set to use pulse mode, then a series of pulses is applied until the vibration is removed. After the vibration is removed, movement can be performed using a standard input shaper. If move mode is selected and the vibration is above the level that can be canceled by the adaptive start, then pulses are applied until the vibration is manageable. If the adaptive start can cancel the vibration, then it is performed and then the motion is stopped using a standard input shaper ( $\mathrm{ZV}$ in this case). As discussed earlier, if the stop command occurs during the start, then the quick stop condition is triggered.

The control system was implemented on the Siemens 314C2DP S7-300 Family PLC driving the portable bridge crane shown in Fig. 1. The crane is largly used for education and experimentation involving input shaping. It is equipped with a simple real time algorithm to perform the convolution required for normal input shaping techniques. The algorithm can be described as sets of gains and delays. Each set corresponds to an impulse in the input shaper. The command signal is fed through the gain-delay sets and the shaped command is formed by summing the resulting signals. The algorithm employs a circular buffer to store the delayed command values each cycle of the control loop. The crane and its input shaping implementation are described further by Lawrence and Singhose [13]. This input shaping implementation was expanded upon for this control system. The control loop containing the input shaping, pulse, and move algorithms was implemented using a temporized interrupt routine with a period of $20 \mathrm{~ms}$.

The pulse and adaptive start algorithms are very similar in structure; they both contain the three sections shown in Fig. 8 and only differ in the third. The direction input section waits for the user to press either the left/right or the up/down buttons. After receiving input, the PLC calculates the frequency and begins monitoring the deflection looking for a minimum or maximum swing condition. The frequency is directly calculated from the hoist length of the payload. The maximum amplitude is found

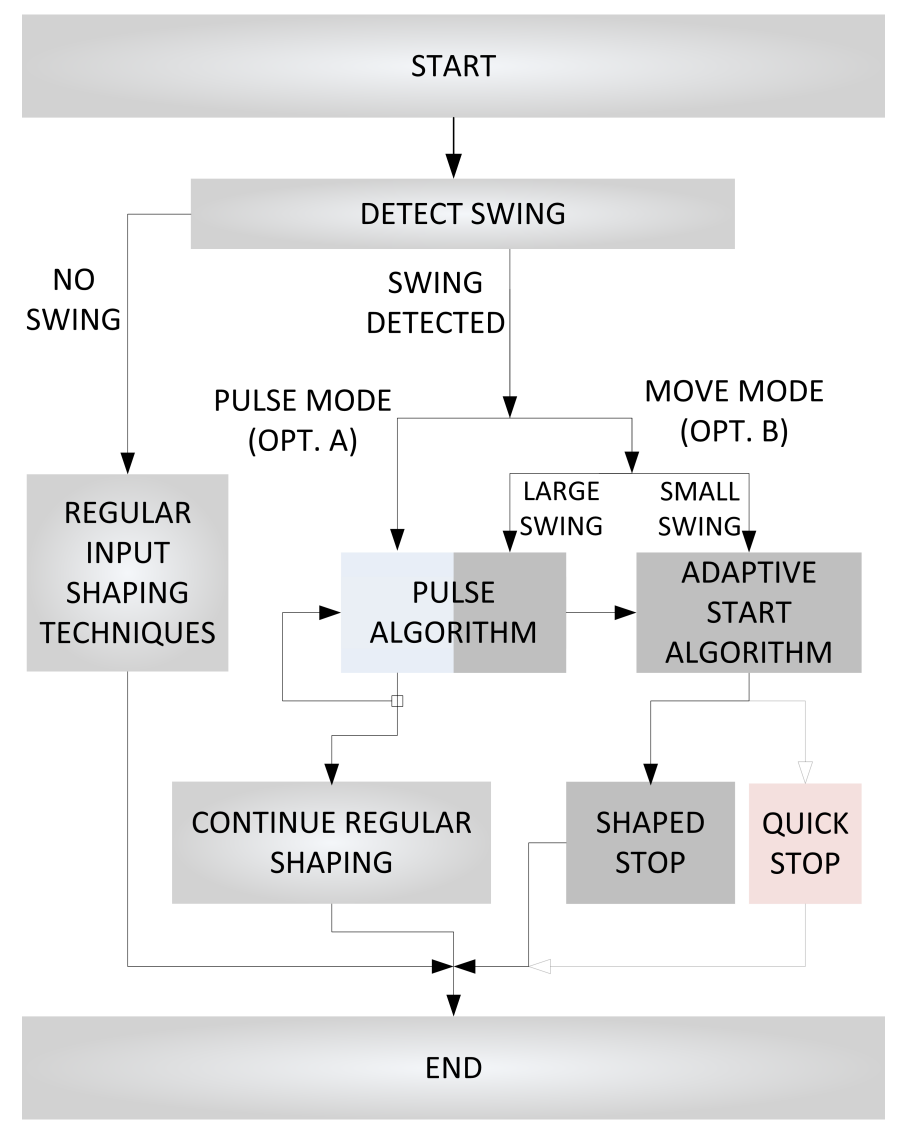

Figure 7. STRUCTURE OF CONTROL SYSTEM

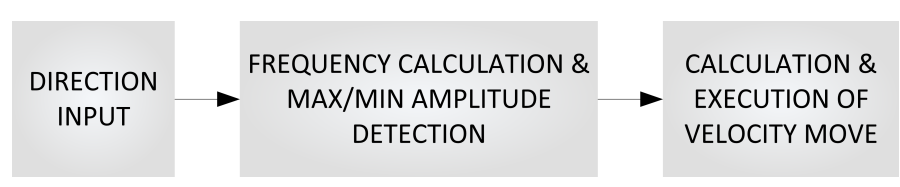

Figure 8. STRUCTURE OF PLC CODE

by continuously sampling the displacement of the payload. A moving average of 3 samples is used to reduce noise and false positives. The first two averages determine if the PLC is looking for a minimum or maximum depending on the slope. A maximum/minimum is detected by a change in slope. The amplitude and time at which the maximum/minimum occurs are noted. Once a maximum/minimum is located, the velocity impulses are calculated.

In the pulse algorithm, a velocity pulse that will induce the correct canceling deflection is then calculated from Eq. 11 and applied as shown in the vector diagram in Fig. 3. PLC counters are used to time the wait before the pulse and its duration. The phase delay discussed earlier is factored into the timing of the impulses to compensate for the rise time of the velocity step response.

In the move algorithm, the velocity impulses are calculated using Eq. 6 and then convolved with a unit step. The veloc- 
ity profile is then executed using the PLC counters. The wait counter times the delay till the first step in velocity and the duration counter times the delay till the step to full velocity. In order to provide the shaped stop functionality of the move mode, a ZV shaper is convolved in real time with the velocity input in the background. During the adaptive start algorithm, the outputs from the shaper are ignored. When both the adaptive start algorithm and the shaper specify full velocity, the velocity output switches from the algorithm to the shaper. Due to this implementation, the quick stop condition had to be handled separately. If a quick stop is required, then the output velocity is set to zero and the wait and duration timers are set such that impulses $\left(A_{3}, t_{3}\right)$ and $\left(A_{4}, t_{4}\right)$ in Fig. 6 can be executed as a velocity pulse.

\section{EXPERIMENTAL CONTROLLER PERFORMANCE Pulse Mode}

When initial oscillations are above a certain threshold (determined by the motors maximum velocity, $V_{\max }$ ), multiple pulses are required to cancel the initial oscillation. This is shown in Fig. 9. With $V_{\max }=0.243 \mathrm{~m} / \mathrm{s}$, the first pulse cancels 81 percent $(197 \mathrm{~mm}$ to $37 \mathrm{~mm})$ of the initial oscillation. A second pulse, appropriately sized to $V=0.074 \mathrm{~m} / \mathrm{s}$, reduces the remaining vibrations further by about 89 percent (from $37 \mathrm{~mm}$ to $4 \mathrm{~mm}$ ). Hence, in such cases 2 or more pulses maybe required to cancel the vibration to a tolerable level. A larger $V_{\max }$ will allow canceling larger initial oscillations with fewer impulses. In the figure, the pulses are spread out to allow for observation of the residual vibration, but the second pulse would general follow quickly after the first, with a short pause to remeasure deflection.

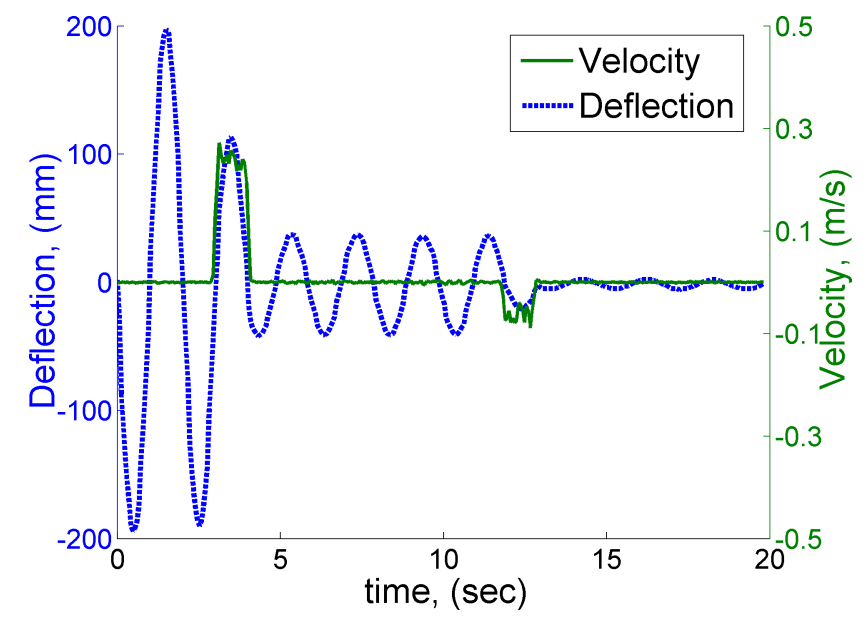

Figure 9. SAMPLE PULSE MODE TRIAL AT 100CM WITH TWO PULSES

Verification of pulse mode performance subject to changes in initial oscillation amplitudes is shown in Fig. 10. Multiple trials of different payload suspension lengths were tested to

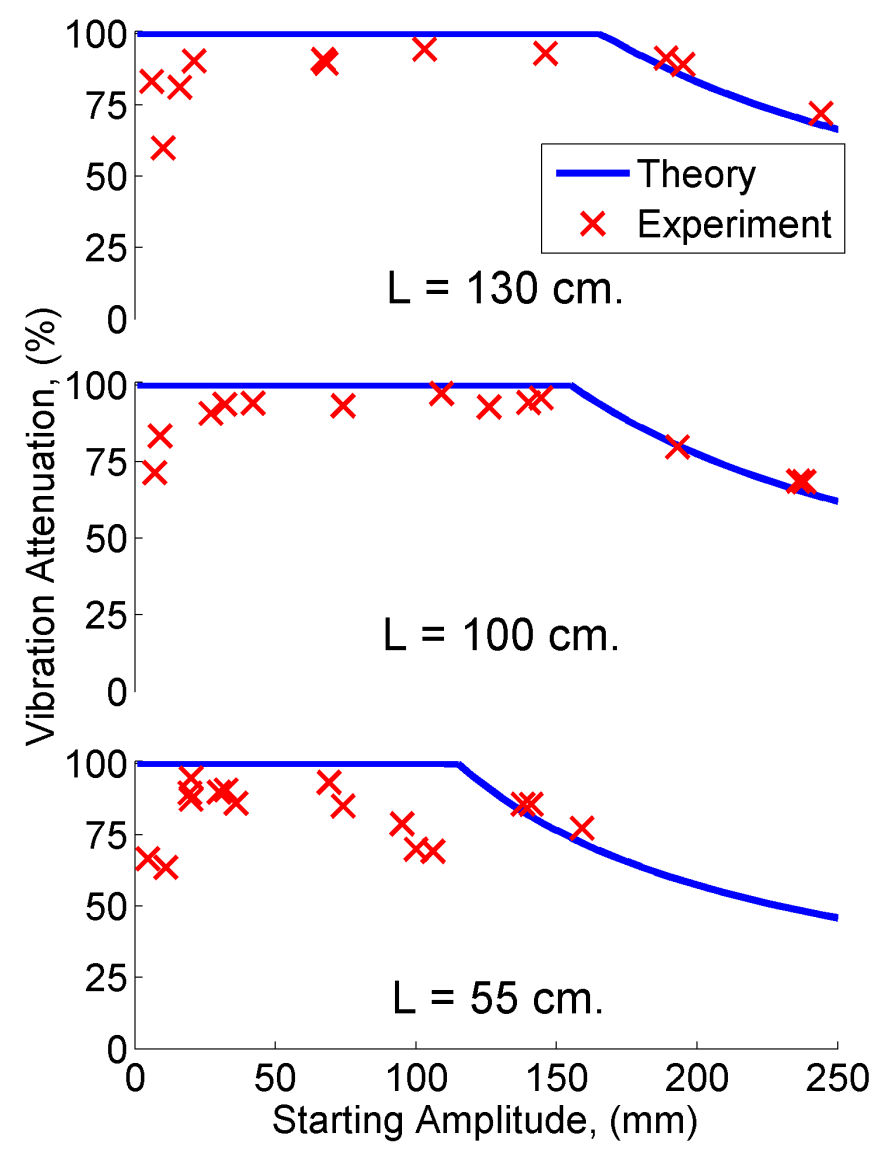

Figure 10. EXPERIMENTAL CONTROLLER VERIFICATION: VARIATIONS IN STARTING OSCILLATION AMPLITUDES

measure performance within the $55-130 \mathrm{~cm}$ range. Theoretical curves, calculated with Eq. 11 by using $V_{\max }=0.243 \mathrm{~m} / \mathrm{s}$, clearly show the cutoff oscillation amplitude in each plot, after which more than 1 pulse is needed to completely cancel the initial oscillation. With the exception of very small starting amplitudes $(0-10 \mathrm{~mm})$, a single pulse results in a relatively consistent attenuation greater than 90 percent of the initial oscillation within the cutoff range. The attenuation did not reach 100 percent due to the estimated rise time adjustment and camera amplitude measurement errors. During experimentation the maximum and minimum detected amplitudes differed by as much as $10 \mathrm{~mm}$. This offset was not consistent and varied between runs. Depending on which was read, the result could be an under or over-sized pulse that would leave some vibration. This measurement offset likely became more significant at the smaller starting amplitudes, resulting in the attenuation drop off seen in the $0-10 \mathrm{~mm}$ range. The more varied performance at the $L=55 \mathrm{~cm}$ range occurs because the camera reading became more unreliable as payload length was shortened. 


\section{Move Mode}

An example trial that demonstrates the move mode is shown in Fig. 11. In the plot the adaptive start and ZV shaped stop sections can be seen. The first two steps in velocity are the adaptive start. After the start has occurred, it can be seen that the initial oscillation is greatly reduced. The second set of steps is the $\mathrm{ZV}$ shaped stop. The $\mathrm{ZV}$ is actively calculated for the estimated period. In this trial the shaper is not perfectly designed, and thus induces some small oscillation during the stop. The remaining vibrations are still smaller by 95 percent. In the plot it is also notable that the residual vibration does not occur about 0 . This is an example of the measurement offset that occurs with any overhead camera.

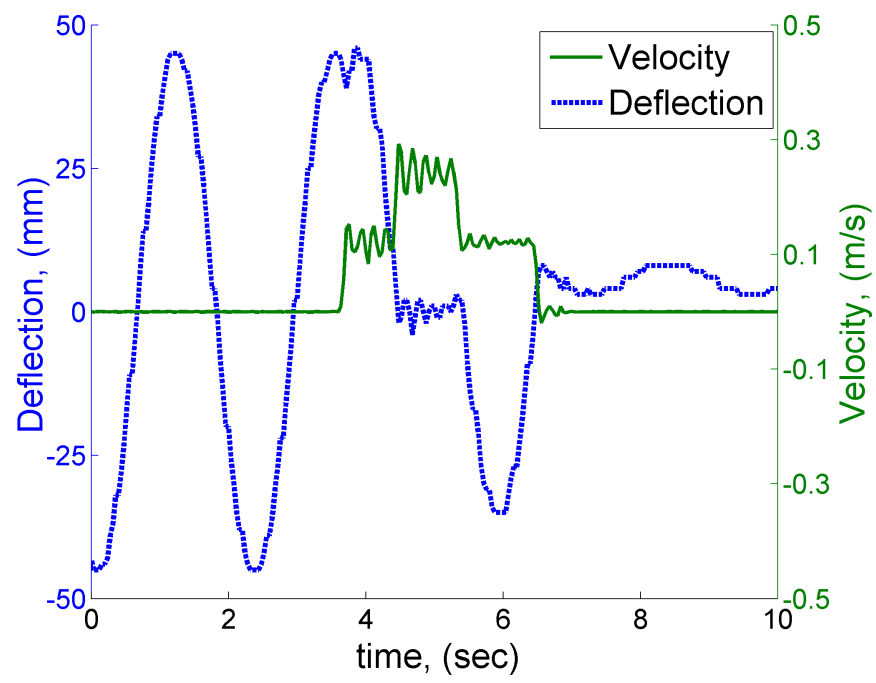

Figure 11. MOVE MODE SAMPLE TRIAL AT LENGTH 130CM

Move mode performance at different initial oscillation amplitudes is shown in Fig. 12. As with the pulse mode, multiple trials at 3 different payload suspension lengths were performed to examine performance across the $55-130 \mathrm{~cm}$ range. The theoretical curves were generated using the fact that the adaptive start converges to a single impulse for large initial swings. The maximum swing that could be canceled was found using Eq. 11 and $V_{\max }=0.243 \mathrm{~m} / \mathrm{s}$. The cutoff starting amplitude for the adaptive start is half that of the pulse because of its convergence to a single impulse. Across the three suspension lengths, the move mode was generally able to achieve attenuation levels of more than 75 percent. Like the pulse mode, performance fell off at smaller starting amplitudes and shorter suspension lengths.

\section{ROBUSTNESS ANALYSIS OF CONTROL SYSTEM}

The residual vibration of a series of impulses is [5]:

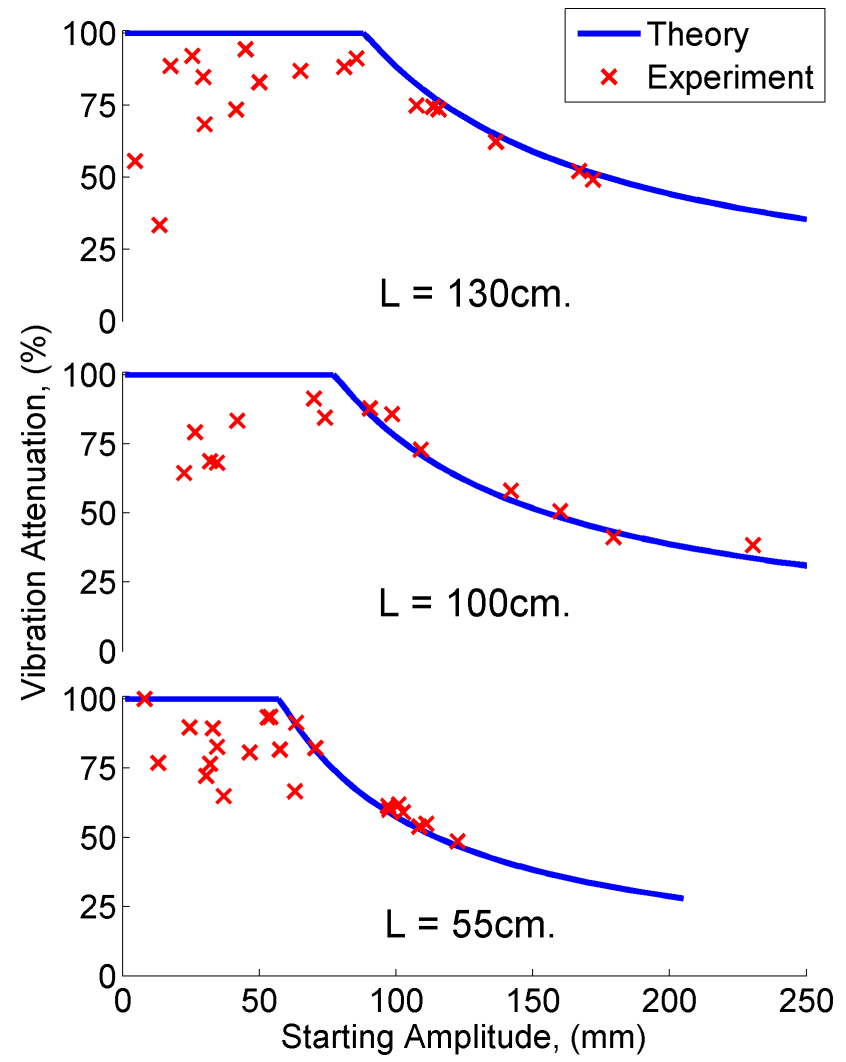

Figure 12. MOVE MODE LENGTH ROBUSTNESS TESTS

$$
V\left(\omega_{n}, \zeta\right)=e^{-\zeta \omega_{n} t_{n}} \sqrt{C^{2}\left(\omega_{n}, \zeta\right)+S^{2}\left(\omega_{n}, \zeta\right)}
$$

where,

$$
\begin{array}{r}
C\left(\omega_{n}, \zeta\right)=\sum_{i=1}^{n} A_{i} e^{\zeta \omega_{n} t_{i}} \cos \left(\omega_{d} t_{i}\right) \\
S\left(\omega_{n}, \zeta\right)=\sum_{i=1}^{n} A_{i} e^{\zeta \omega_{n} t_{i}} \sin \left(\omega_{d} t_{i}\right)
\end{array}
$$

This equation is normalized with respect to the vibrations caused by a unity impulse. This equation may be used to analyze the robustness of these controllers if the initial impulse is assumed to be unity and included in the series of impulses. This changes the result of Eq. 12 to the residual vibration with respect to the initial vibration.

\section{Pulse Mode}

Robustness tests were performed for the pulse mode using a shaper given by: 


$$
\left[\begin{array}{c}
A_{i} \\
t_{i}
\end{array}\right]=\left[\begin{array}{ccc}
1 & 0.5 & -0.5 \\
0 & 0.5 T_{m} & T_{m}
\end{array}\right]
$$

where, $T_{m}$ is the model period.

The theoretical residual vibration with respect to frequency estimation error with no damping is shown in Fig. 13, where $\omega_{a}$ is the actual frequency and $\omega_{m}$ is the model frequency. This demonstrates the robustness to conditions where the payload length is changed after the action has been calculated, but before it has been executed. This plot shows that it is possible for the controller to make vibrations worse by 159 percent of the initial value if the normalized frequency is above or below 1 by 0.54 . The controller will however reduce vibration by some amount if the frequency is between 0.77 and 1.228 , meaning the vibration would eventually be removed after several pulses.

The theoretical residual vibration with respect to the estimation of the initial swing amplitude is shown in Fig. 14, where $A_{a}$ is the actual and $A_{m}$ is the measured amplitude. This was calculated by leaving impulse $A_{0}$ constant while varying the size of $A_{1}$ and $A_{2}$ as they would change with measured amplitude. The plot shows that there is a direct relationship between the amplitude ratio difference from 1 and the residual vibration. This shows how important the amplitude estimation is to canceling the oscillation.

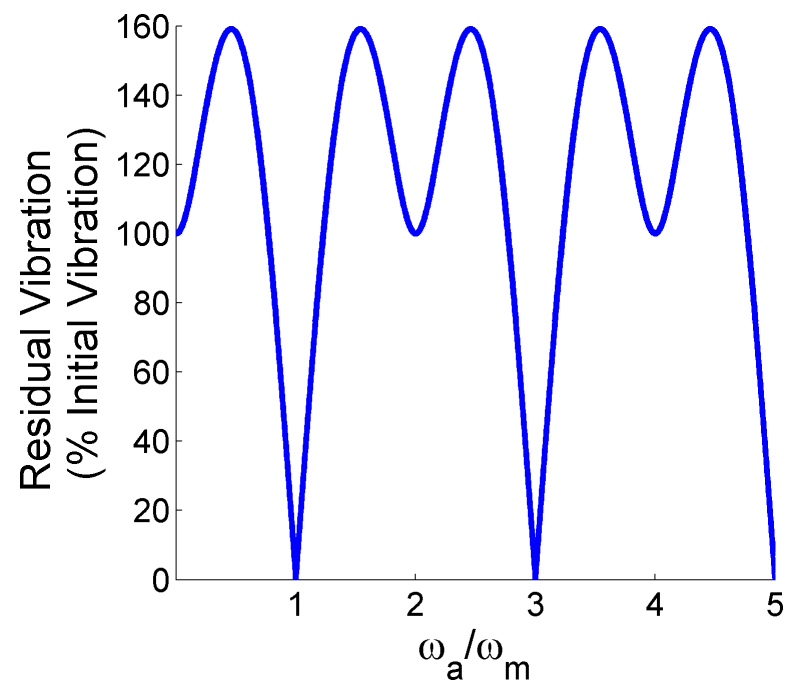

Figure 13. RESIDUAL VIBRATION VS FREQUENCY

This controller uses the payload suspension length to compute both the frequency and the velocity step size. It is therefore important to examine the robustness to length measurement errors. The effect of length errors on amplitude can be derived as follows: Using Eq. 11 a relationship between the measured and actual initial velocity amplitudes, $A_{m}$ and $A_{0}$ based on the actual and measured lengths, $L_{a}$ and $L_{m}$, can be calculated.

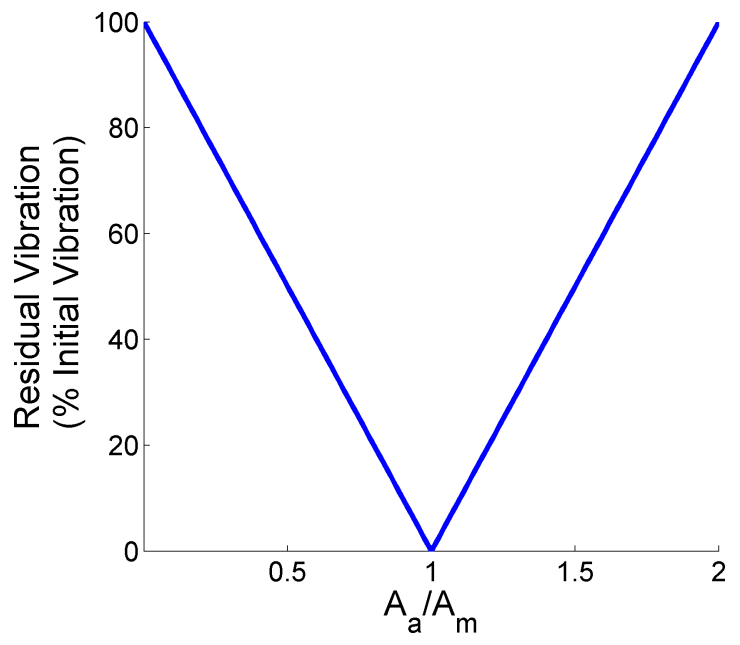

Figure 14. RESIDUAL VIBRATION VS AMPLITUDE

$$
A_{m}=\frac{g}{\omega_{m}} \sin ^{-1}\left(\frac{x_{a}}{L_{m}}\right)
$$

The frequencies can be written in terms of their respective lengths using:

$$
\omega_{n}^{2}=\left(\frac{g}{L}\right)
$$

Therefore the ratio $c$ can be defined as:

$$
c=\frac{\omega_{a}}{\omega_{m}}=\sqrt{\frac{L_{m}}{L_{a}}}
$$

Plugging in for $L_{m}$ and $\omega_{m}$, then assuming that the deflections are much less than the lengths and applying the small angle approximation yields:

$$
A_{m}=\frac{c g}{\omega_{a}} \sin ^{-1}\left(\frac{x_{a}}{c^{2} L_{a}}\right)=\left(\frac{1}{c}\right) A_{a}
$$

The residual vibration can then be calculated in terms of $c$ by changing both the frequency and amplitude of $A_{1}$ and $A_{2}$ as in the amplitude robustness plot. The theoretical residual vibration with respect to length error is shown in Fig. 15 along with experimental data. In the figure it can be seen that the experimental data matches the theoretical curve relatively well especially in the region around 1 . This region is where operation is most likely to occur. The 20 percent insensitivity range is 0.16 , 


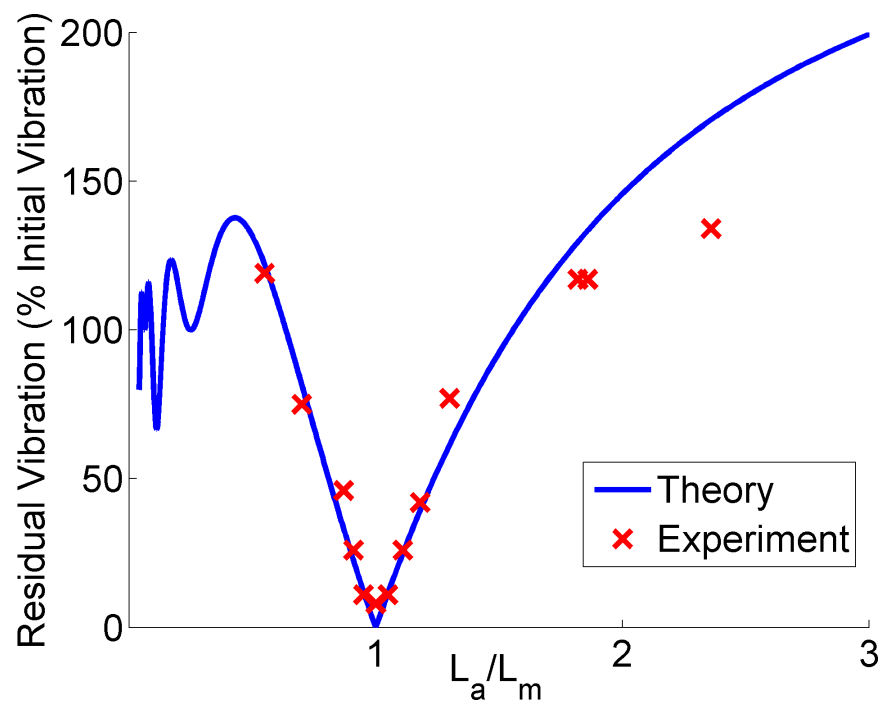

Figure 15. RESIDUAL VIBRATIONS VS. LENGTH ERROR

however, the controller will still yield a reduction in swing for normalized lengths between 0.64 to 1.55 . In this range the swing could eventually be stopped using multiple pulses. It is also important to note that the farther the normalized length is from one, the less likely the situation is to occur on an actual system. Hoist lengths measured by encoders have a precision of about \pm 1 percent. Large errors would most likely be noticed by the crane operator and addressed before causing issues. A large payload may also cause an error in the effective length, however, as mentioned previously the oscillation will still be reduced over a large range.

\section{Move Mode}

The robustness of the move mode was examined using the impulse sequence given by:

$$
\left[\begin{array}{c}
A_{i} \\
t_{i}
\end{array}\right]=\left[\begin{array}{ccc}
1 & 0.5 / A_{0} & 0.5 / A_{0} \\
0 & \frac{\pi-\theta}{2 \pi} T & \frac{\pi+\theta}{2 \pi} T
\end{array}\right]
$$

This is a modified form of Eq. 6 with $A_{R}$ assumed to be 1 and normalized with respect to the initial vibration step velocity $A_{0}$.

The timings of the move mode impulses change with different initial oscillation amplitudes; therefore, the robustness was analyzed by looking at the 20 percent insensitivity range across the range of actual initial step velocity amplitudes. The initial amplitudes were varied from 0 to 1 such that they were less than or equal to the assumed requested velocity of 1 .

The range of $\omega_{a} / \omega_{m}$ that will produce a residual vibration that is 20 percent or less of the initial vibration is shown in Fig. 16 as a function of the ratio of the initial vibration step velocity amplitude $A_{0}$ to the requested velocity $A_{R}$. As with pulse mode, this demonstrates the robustness in situations where the suspension length is changed after calculation, but before execution. In the plot it can be seen that the insensitivity range increases as the initial vibration amplitude increases. The insensitivity reached a maximum of approximately 0.13 . Frequency error has a much larger effect at smaller initial amplitudes, because the impulse amplitudes are tied to the requested velocity. At smaller initial oscillation amplitudes the the requested velocity is much larger and so badly timed impulses are able to cause more vibration. This results in a narrower range that will produce acceptable results.

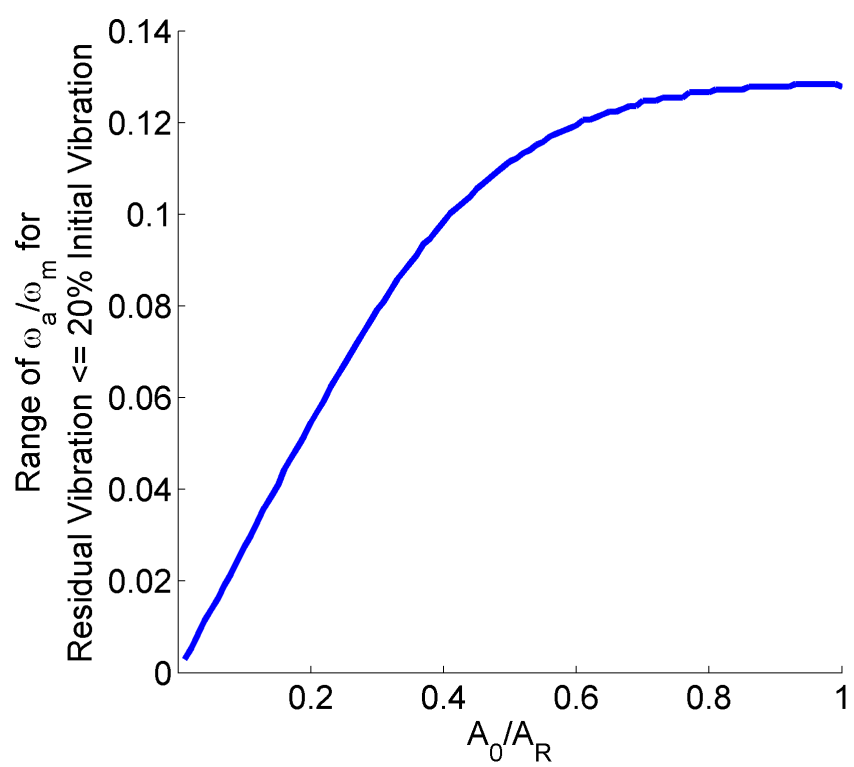

Figure 16. FREQUENCY ROBUSTNESS VS INITIAL VIBRATION STEP VELOCITY

The theoretical residual vibration with respect to amplitude measurement error was also examined for the move mode control system. The results at each initial vibration level were found to be almost identical to the robustness found for the pulse mode, shown in Fig. 14. There was a direct relationship between the error in the amplitude and the residual vibration. This is why the variation seen in Fig. 12 is due to amplitude measurement error.

Like the pulse mode, the move move uses the payload suspension length to calculate the velocity step size that caused the initial vibration and the natural frequency of the system. The most critical aspect that is effected by length measurement errors is the initial vibration velocity amplitude $A_{0}$ as it is used in the calculation of the impulse timings. Using the relationship derived in Eq. 19, the robustness of the move mode to length measurement errors was examined. The range of $L_{a} / L_{m}$ that produces a residual vibration level at or below 20 percent of the initial oscillation amplitude is shown in Fig. 17 as a function of the ratio of $A_{0}$ to $A_{R}$. Again the insensitivity range increases as the ini- 
tial vibration step velocity increased. This is for the same reason discussed for the frequency robustness. The bump that is seen near the velocity ratio of 0.85 is an effect of $A_{0}$ being limited to less than $A_{R}=1$. $A_{0}$ changes with $c$ but at higher starting points it hits the limit faster. The bump is the limit being seen in the interval. In the plot it can be seen that the move mode provides a 20 percent insensitivity level that is higher than the pulse mode's 0.16 over most of the initial oscillation levels.

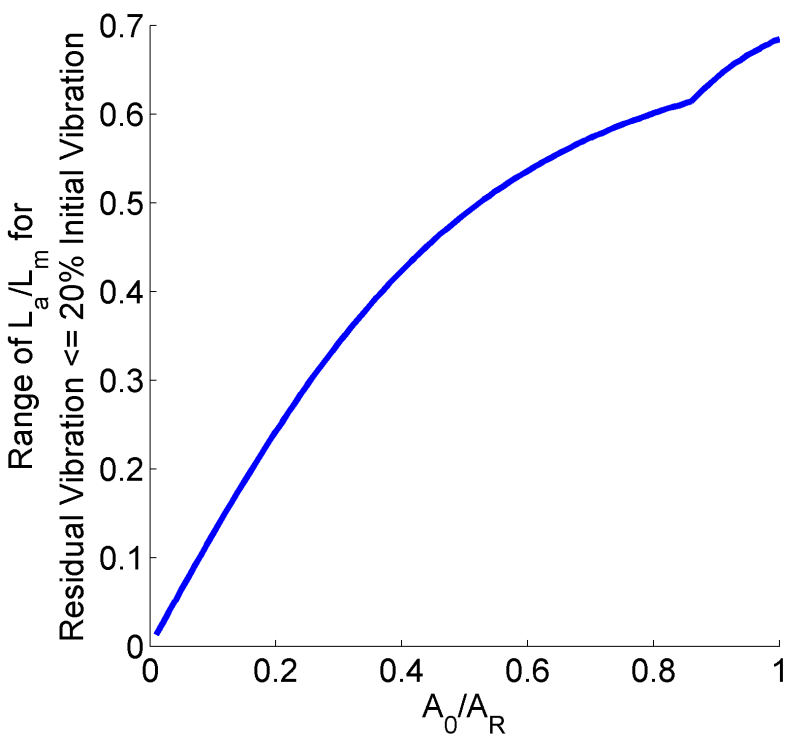

Figure 17. LENGTH ROBUSTNESS VS INITIAL VIBRATION STEP VELOCITY

\section{CONCLUSION}

Two parallel control systems have been developed and shown to work well for reducing initial payload oscillation of bridge cranes. Effective attenuation reached as much as 97 percent of the initial payload swing, resulting in negligible or acceptable final payload swing. Pulse mode experimental results showed that, on average, first pulse attenuation reached above 90 percent, and that most of the initial oscillation amplitudes can be effectively canceled with only 2 successive pulses. Move mode experimental results showed that in the majority of cases attenuation was above 80 percent. In conclusion, the developed controller met all three performance objectives. That is, the controller accurately detected the maximum initial swing amplitude and correctly calculated the swing frequency; was able to effectively cancel out these oscillations; and allowed the crane operator to choose in which direction the cancellation action was executed. Finally, experimental results validated robustness models. It was shown that the controllers produced attenuation of at least 80 percent across a respectable range of frequency, amplitude, and length measurement errors.

\section{ACKNOWLEDGEMENTS}

The authors would like to thank Siemens Energy and Automation and Boeing Research and Technology for their support of this work.

\section{REFERENCES}

[1] William E. Singhose, W. P. S., 2007. Command Generation for Dynamic Systems. LuLu, Atlanta.

[2] Starr, G. P., 1994. "Swing-free transport of suspended objects with a path-controlled robot manipulator". J. of $D y$ namic Systems, Measurement and Control, 107, pp. 97100.

[3] Strip, D. R., 1989. "Swing-free transport of suspended objects: A general treatment". IEEE Trans. on Robotics and Automation, 5(2), pp. 234-236.

[4] Parker, G. G., Groom, K., Hurtado, J. E., Feddema, J., Robinett, R. D., and Leban, F., 1999. "Experimental verification of a command shaping boom crane control system". In American Control Conference, pp. 86-90.

[5] Sorensen, K., Singhose, W., and Dickerson, S., 2007. "A controller enabling precise positioning and sway reduction in bridge and gantry cranes". Control Engineering Practice, 15(7), pp. 825-837.

[6] Hekman, K. A., and Singhose, W. E., 2007. "Feedback control system for suppressing crane oscillations with on-off motors". Int. Journal of Control, Automation, and Systems, 5(3), pp. 223-233.

[7] Kim, D., and Singhose, W. "Performance studies of human operators driving double-pendulum bridge cranes". Control Engineering Practice, 18, June.

[8] Vaughan, J., Kim, D., and Singhose, W., 2010. "Control of tower cranes with double-pendulum dynamics". IEEE Trans. on Control Systems Technology, 18(6), pp. 13451358.

[9] Lewis, D., Parker, G. G., Driessen, B., and Robinett, R. D., 1998. "Command shaping control of an operator-inthe-loop boom crane". In American Control Conference, pp. 2643-2647.

[10] Singer, N. C., and Seering, W. P., 1989. "Design and comparison of command shaping methods for controlling residual vibration". In IEEE International Conference on Robotics and Automation, Vol. 2, pp. 888-893.

[11] Singhose, W. E., Seering, W. P., and Singer, N. C., 1990. "Shaping inputs to reduce vibration: A vector diagram approach". In IEEE International Conference on Robotics and Automation, Vol. 2, pp. 922-927.

[12] Smith, O. J. M., 1957. "Posicast control of damped oscillatory systems". pp. 1249-1255.

[13] Lawerence, J., and Singhose, W., 2005. "Design of minicrane for education and research". In 6th Int. Conference on Research and Education in Mechatronics. 